\title{
Numerical Evaluation of Multiple Integrals I
}

Introduction. Several specific methods for numerical evaluation of integrals over higher dimensional regions have been proposed. James Clerk-Maxwell [1] proposed the formulas for the rectangle and the rectangular parallelopipedon in 1877. Appell, Burnside, Ionescue, and Mineur have developed special formulas for planar regions. Tyler [2] recently gave formulas for rectangles, parabolic regions, cubes, and for the hypercubes. Others have developed formulas primarily for rectangular regions based on the formulas for the line.

Although the original draft of this paper antedates their results, Hammer, Marlowe and Stroud [3] have given an inductive method for numerical evaluation of integrals precise for $k$ th degree polynomials over the $n$-simplex. They also show how to obtain formulas for cones based on regions for which integration formulas are given. Certain affinely symmetrical formulas for triangle and tetrahedron are given in their paper. In a sequel Hammer and Stroud [4] gave affinely symmetrical formulas precise for the quadratic and cubic polynomials over the $n$-simplex. W. H. Peirce [5] in his doctoral dissertation has given numerical integration formulas over the circular annulus precise for arbitrarily high degree polynomials.

Despite the variety of results and their particular interest, the theory of numerical integration in higher dimensions is in a very crude state of development in comparison with numerical integration of functions of one variable. For this reason we give here a few theorems which are actually quite simple but which evidently have escaped the awareness of most research workers. These theorems form a partial basis for development of classical type integration formulas.

The sole numerical integration device with claims to somewhat universal applicability is that called the Monte Carlo method which has been extended and popularized by S. M. Ulam and John von Neumann. Granted the presence of a suitable sequence of "random" digits or the means of generating such, the Monte Carlo methods can be applied to a large variety of problems by using high-speed digital computers. It has been stated as an advantage of Monte Carlo methods that the number of evaluation points for, say, 5 percent error does not mount as rapidly for higher dimensions as it does for classical formulas. We shall show (as Tyler, and Hammer and Stroud have shown in other cases) that the evaluation points for classical type formulas can conceivably be kept down very well. However, the problem of determining points and weights in these circumstances is a problem in the theory of equations which is difficult inherently and also because of the great variety of functions and regions of conceivable interest. In this direction the Monte Carlo method is less sensitive to the deviations among regions.

A Basic Theorem for Numerical Integration. In this section we state a theorem which greatly extends the usefulness of particular integration formulas when they are available. We limit the discussion to integration formulas of the form

$$
\int_{R} w(x) f(x) d x \doteq \sum a_{j} f\left(x_{j}\right)
$$


where $R$ is an $n$-dimensional region in Euclidean $n$-space, $E_{n}, x$ is a point vector in $E_{n}, a_{1}, \cdots, a_{m}$ are numbers (real or complex) and evaluation points $x_{1}, \cdots, x_{m}$ are in the domain of $f$. In particular, $R$ will be taken as the closure of a bounded open set in $E_{n}$ and the weight function $w(x)$ and $f$ are continuous on $R$. That these conditions may be relaxed will not concern us. The $d x$ indicates a volume element in $E_{n}$.

For each relevant $f$ and a given formula (1) there is the associated error, a number $E(f)$, which we define as

$$
E(f)=\sum a_{j} f\left(x_{j}\right)-\int_{R} w(x) f(x) d x .
$$

Let $S$ be another region in $E_{n}$ such that there exists a transformation $J$ with continuous.nonvanishing Jacobian which maps $R$ onto $S$. We write $y=J x$ for $x \in R$. Then if $g(y)$ is defined and continuous on $S$ we may write

$$
\int_{S} w(y) g(y) d y=\int_{R} w(y) g(y)|J| d x,
$$

where in the integrand on the right we have $y=J x$ and $|J|$ is the absolute value of the Jacobian which may also be indicated as $\partial y / \partial x$. Now if we have an integration formula (1) for $R$ and the choice $w(x)=w_{1}(y)$, then with $f(x)$ replaced in (2) by $|J| g(y)$ we have

$$
E(|J| g)=\sum a_{j}\left|J_{j}\right| g\left(y_{j}\right)-\int_{R} w_{1}(y) g(y)|J| d x,
$$

where $y_{j}=J x_{j}$ and $\left|J_{j}\right|$ is the value of $|J|$ at $x_{j}$.

THEOREM 1. Writing

$$
E(S, g)=\sum b_{j} g\left(y_{j}\right)-\int_{S} w_{1}(y) g(y) d y,
$$

where $b_{j}=a_{j}\left|J_{j}\right|$, then $E(S, g)=E(|J| g)$ and hence from every formula of type (1) for $R$ there is a corresponding formula of the same type for $S$ with error functional $E(S, g)$.

Corollary 1.1. If $|J|$ is constant on $R$ then $E(S, g)=|J| E(g)$ and hence if $E(g)$ vanishes for a function $g$ so does $E(S, g)$. In particular, if the transformation $J$ is a nonsingular affine transformation, $|J|$ is constant.

COROLlaRY 1.2. Identifying $w(x)$ with $w_{1}(y)|J|, y=J x$ and $f(x)$ with $g(y)$, assuming a formula (1), gives from equation (2)

$$
E(S, g)=E(g)=\sum a_{j} g\left(y_{j}\right)-\int_{S} w_{1}(y) g(y) d y .
$$

Theorem 1 and the two corollaries make it possible to generate integration formulas for a variety of regions. The simplest and the most important case arises 
when $J$ is an affine transformation (Corollary 1.1). Thus certain numerical integration formulas over a particular sphere precise for polynomials of at most degree $k$ give immediately integration formulas for any ellipsoid precise for polynomials of at most degree $k$.

Cartesian Product Regions. Let $R$ be the Cartesian product of two regions $R_{1}$ and $R_{2}$ in lower dimensional Euclidean spaces. Let us designate $y \in R_{1}, z \epsilon R_{2}$ so that every $x \in R$ can be written $(y, z)$. Suppose there are numerical integration formulas given of type (1) for weight functions $w_{1}(y)$ and $w_{2}(z)$, defined on $R_{1}$ and $R_{2}$ respectively, so that the error functionals may be written

$$
\begin{aligned}
& E\left(R_{1}, f_{1}\right)=\sum a_{i} f_{1}\left(y_{i}\right)-\int_{R_{1}} w_{1}(y) f_{1}(y) d y \\
& E\left(R_{2}, f_{2}\right)=\sum b_{j} f_{2}\left(z_{j}\right)-\int_{R_{2}} w_{2}(z) f_{2}(z) d z .
\end{aligned}
$$

Then we ask for a numerical integration formula over $R=R_{1} \times R_{2}$ with weight function $w_{1}(y) w_{2}(z)$. We have, by using (1) and (2),

THEOREM 2. Let $f(x)=f(y, z)$ be a function defined and continuous on $R=R_{1}$ $\times R_{2}$. Then if we define

$$
E(R, f)=\sum \sum a_{i} b_{j} f\left(y_{i}, z_{j}\right)-\int_{R_{1}} \int_{R_{2}} w_{1}(y) w_{2}(z) f(y, z) d y d z,
$$

we have

$$
\begin{aligned}
E(R, f) & =\sum b_{j} E\left[R_{1}, f\left(y, z_{j}\right)\right]+\int_{R_{1}} w_{1}(y) E\left[R_{2}, f(y, z)\right] d y \\
& =\sum a_{i} E\left[R_{2}, f\left(y_{i}, z\right)\right]+\int_{R_{2}} w_{2}(z) E\left[R_{1}, f(y, z)\right] d z .
\end{aligned}
$$

CoRollary 2.1. If $f(y, z)$ is a function such that $E\left[R_{1}, f(y, z)\right]=0$ for each $z \in R_{2}$ and $E\left[R_{2}, f(y, z)\right]=0$ for each $y \in R_{1}$ then $E(R, f)=0$ and the formula (3) of Theorem 2 is exact for $f$.

If $F_{1}$ is a class of functions defined on $R_{1}$, and $F_{2}$ is a class of functions defined on $R_{2}$, then the Cartesian product class, $F=F_{1} \times F_{2}$, of functions defined on $R_{1} \times R_{2}$ is the class of all functions $f(y, z)$ such that $f(y, z) \epsilon F_{1}$ for each $z \in R_{2}$ and $f(y, z) \in F_{2}$ for each $y \in R_{1}$.

CoRollary 2.2. If $E\left(R_{1}, f_{1}\right)=0$ for $f_{1} \epsilon F_{1}$ and $E\left(R_{2}, f_{2}\right)=0$ for $f_{2} \epsilon F_{2}$ then necessarily $E(R, f)=0$ for every $f \in F_{1} \times F_{2}$, where the error functionals are defined by (1), (2), (3). In other words, the numerical integration formula in (3) is necessarily exact for the Cartesian product of the classes of functions for which (1) and (2) are exact.

CoRollary 2.3. If classes of functions $F$ and $G$ respectively defined on $R_{1}$ and $R_{2}$ are representable as all linear combinations of basis sets of functions $f_{1}, \cdots, f_{p}$ and $g_{1}, \ldots, g_{q}$ respectively then $F \times G$ is the set of all functions with $\left(f_{i} g_{j}\right)$ as basis.

Proof: If $h=\sum_{i} \sum_{j} c_{i j} f_{i} g_{j}$ where the $c_{i j}$ are constants, then $h \in F \times G$. To show that $h \in F \times G$ implies $h$ is a linear combination of the $f_{i} g_{j}$, we observe that 
$h=\sum_{i} a_{i} f_{i}=\sum_{j} b_{j} g_{j}$ where the $a_{i}$ are unique functions on $R_{2}$, and $b_{j}$ are unique functions on $R_{1}$. Let $y_{1}, y_{2}, \cdots, y_{p}$ be the points in $R_{1}$ such that the determinant $\left|f_{i}\left(y_{k}\right)\right|$ is not zero. Then we have with $b_{j k}$ the value of $b_{j}$ at $y_{k}$

$$
\sum_{i} a_{i} f_{i}\left(y_{k}\right)=\sum_{j} b_{j k} g_{i} \quad k=1, \cdots, p
$$

which are identities on $R_{2}$. Solving for $a_{1}, \cdots, a_{p}$ we find $a_{i}=\sum_{j} c_{i j} g_{j}$ where $c_{i j}$ are constants and are unique. Hence $h=\sum_{i} \sum_{j} c_{i j} f_{i} g_{j}$.

The usefulness of the Cartesian product principle is extensive. However, even here there must be limitations. Thus over the hypercube in $E_{n}$ the generation of a formula based on Cartesian powers of the line segment formulas with $m$ points gives $m^{n}$ points. If $n$ is large and $m \geq 2$, this will involve a large number of points of evaluation.

Examples. Gauss' four-point formula on the line is exact for at most 7 th degree polynomials in one variable. Hence the 16-point formula

$$
\sum_{1}^{4} \sum_{1}^{4} a_{i} a_{j} f\left(x_{i}, x_{j}\right) \doteq \iint_{R} f(x, y) d x d y
$$

where $a_{i}$ are the Gauss weights, $R$ is the square, and $x_{i}$ are the zeros of the Legendre polynomial, is exact for $f(x, y)=\sum \sum c_{i j} x^{i} y^{j} 0 \leq i \leq 7,0 \leq j \leq 7$ since this class of polynomials is the Cartesian square of the class of seventh degree polynomials in one variable. Observe that this class contains all seventh degree polynomials and additional polynomials since $i$ and $j$ range independently from 0 to 7 . Similarly formulas may be developed for the hypercubes.

Laguerre's 3-point formula for weight function $e^{-x}$, interval $0, \infty$ holds for all fifth degree polynomials. Hence

$$
\sum_{1}^{3} \sum_{1}^{3} a_{i} a_{j} f\left(x_{i}, x_{j}\right) \doteq \int_{0}^{\infty} \int_{0}^{\infty} e^{-(x+y)} f(x, y) d x d y
$$

holds exactly for every $f$ of form $\sum_{i \leq 5} \sum_{j \leq 5} c_{i j} x^{i} y^{j}$. Thus we obtain a formula for the quadrant. A linear transformation will give a formula for an infinite planar sector. The $x_{i}$ are the zeros of the Laguerre polynomials. Again these may be used to obtain formulas for octants, etc., in higher dimensions.

Combinations of Gauss formulas and Laguerre formulas give formulas for the planar half-strip, and combinations of Hermite's and Laguerre's will give formulas for the half-plane. A formula for a planar region generates a formula for the cylinder based on this region. In scattering and potential problems Cartesian product regions which are powers or products of 3-dimensional regions are common. Thus formulas for each region are useful for generating others.

P. Davis of the National Bureau of Standards wrote of an example he and P. Rabinowitz estimated and which has since been published [6]. This example is to integrate exp $\left(x_{1} x_{2} x_{3} x_{4}\right)$ over the unit cube in 4 -space. We calculated an esti- 
mate using the fourth "power" of the Gauss two-point formula, and obtained with these 16 points half the error reported by Davis and Rabinowitz using $2^{15}=32,768$ points and a Monte Carlo method. Since the cost is roughly proportional to the number of points, it is seen that the classical type formula is here much more efficient. Of course this function is particularly fortunate for Cartesian power methods since it is expandable in powers of $x_{1} x_{2} x_{3} x_{4}$.

Particular Formulas for Symmetrical Regions. In a sequel to this paper we will publish a table of formulas for the regions we have considered, in detail. However, we prefer to define symmetrical regions and correspondingly symmetrical formulas in this paper and to illustrate applications in one type of case since consideration of these problems has led to a variety of solved and unsolved related ones.

Rather than introduce specialized terms we shall say a region $R$ in $n$-space is symmetrical if : whenever it contains a point $x$, it also contains all points obtained from $x$ by interchanging coordinates and changing signs of coordinates. Thus in general with a point $x$ there are required $2^{n} n$ ! points (including $x$ ). A symmetrical region $R$ is invariant under linear transformations with matrix containing exactly one non-zero element in each row and column, that non-zero element being either +1 or -1 . In evaluating integrals of monomials over $R$, symmetry as defined, saves effort as indicated in the following theorem.

THEOREM 3. The integral over a symmetrical region $\mathrm{R}$ of any monomial containing an odd power is zero, the integral of a product of even powers depends only on the exponents and not on their order.

The proof of this theorem is direct in view of the supposed symmetry of $R$. Now if we impose a requirement of symmetry on a numerical integration formula we find that it is possible to compute useful formulas for these regions which have not heretofore been available. A numerical integration formula is symmetrical if the set of evaluation points is decomposable into symmetrical sets of points, each point in a symmetrical set having the same weight as the others.

THEOREM 4. Let $\sum a_{j} f\left(x_{j}\right)$ be a symmetrical sum formula approximating the integral $\int_{R} f(x) d x$ for $R$ symmetrical. In order that $\sum a_{j} f\left(x_{j}\right)-\int_{R} f(x) d x=0$ for every polynomial of degree at most $2 k+1$ it is necessary and sufficient that the formula hold exactly for all monomials $f$ of form

where

$$
t_{1}^{2 k_{1}} \cdots t_{n}^{2 k_{n}}
$$

$$
k_{1} \geq k_{2} \cdots \geq k_{n} \geq 0, \quad k_{1}+\cdots+k_{n} \leq k \text {. }
$$

We illustrate the application of Theorem 4 by considering symmetrical regions in 3-space and indicating a method of obtaining a formula involving at most 34 points holding exactly for 7 th degree polynomials. The exact formula is given for a cube. Now in 3-space an arbitrary selection of a point would involve 47 others with the same weight. Hence we select points for which the symmetry requirement does not yield as many points. In considering the problem we will use $(x, y, z)$ as coordinates. From Theorem 4, we then solve equations only resulting from 
asking that the symmetrical formula hold exactly for the following seven functions: $1, x^{2}, x^{4}, x^{2} y^{2}, x^{6}, x^{4} y^{2}, x^{2} y^{2} z^{2}$ in order to achieve a formula exact for the general seven-degree polynomial. For seventh degree in $n$ variables with a symmetrical formula over a symmetrical region, the same seven functions suffice. Now let the integrals of the seven functions given over $R$ be $I_{0}, I_{2}, I_{4}, I_{22}, I_{6}, I_{42}$, $I_{222}$, respectively. We choose points and weights as follows: $\left(x_{1}, 0,0\right), a_{1}(6)$; $\left(x_{2}, x_{2}, 0\right), a_{2}(12) ;\left(x_{3}, x_{3}, x_{3}\right), a_{3}(8) ;\left(x_{4}, x_{4}, x_{4}\right), a_{4},(8)$. The integer in parenthesis indicates the number of points in the formula generated by the required symmetry if the indicated letter coordinate is not zero. Since only squares of unknown coordinates appear we let $u_{1}=x_{1}{ }^{2}, u_{2}=x_{2}{ }^{2}, u_{3}=x_{3}{ }^{2}$ and $u_{4}=x_{4}{ }^{2}$.

The equations then are:

$$
\begin{aligned}
6 a_{1}+12 a_{2}+8 a_{3}+8 a_{4} & =I_{0} & & f=1 \\
2 a_{1} u_{1}+8 a_{2} u_{2}+8 a_{3} u_{3}+8 a_{4} u_{4} & =I_{2} & & f=x^{2} \\
2 a_{1} u_{2}^{2}+8 a_{2} u_{2}^{2}+8 a_{3} u_{3}^{2}+8 a_{4} u_{4}^{2} & =I_{4} & & f=x^{4} \\
4 a_{2} u_{2}^{2}+8 a_{3} u_{3}^{2}+8 a_{4} u_{4}^{2} & =I_{22} & & f=x^{2} y^{2} \\
2 a_{1} u_{1}^{3}+8 a_{2} u_{2}^{3}+8 a_{3} u_{3}^{3}+8 a_{4} u_{4}^{3} & =I_{6} & & f=x^{6} \\
4 a_{2} u_{2}^{3}+8 a_{3} u_{3}^{3}+8 a_{4} u_{4}^{3} & =I_{42} & & f=x^{4} y^{2} \\
8 a_{3} u_{3}^{3}+8 a_{4} u_{4}^{3} & =I_{222} & & f=x^{2} y^{2} z^{2} .
\end{aligned}
$$

It will be observed that the set constitutes seven equations in eight unknowns so that we may hope for one free choice of another equation. To indicate the solution of the equation we now form (6)-(7) and $(5)-2(6)+(7)$ to get, respectively,

$$
\begin{aligned}
& 4 a_{2} u_{2}^{3}=I_{42}-I_{222} \\
& 2 a_{1} u_{1}^{3}=I_{6}-2 I_{42}+I_{222} .
\end{aligned}
$$

Hence if a solution to the equation system exists and the numbers on the right of (8) and (9) are not zero then neither $u_{1}$ nor $u_{2}$ is zero and hence

$$
\begin{aligned}
& 4 a_{2}=\left(I_{42}-I_{222}\right) / u_{2}{ }^{3} \\
& 2 a_{1}=\left(I_{6}-2 I_{42}+I_{222}\right) / u_{1}{ }^{3} .
\end{aligned}
$$

Now from (3)-(4) we find

$$
2 a_{1} u_{1}^{2}+4 a_{2} u_{2}^{2}=I_{4}-I_{22},
$$

and on substituting from (10) and (11) in (12) we have

$$
\frac{I_{6}-2 I_{42}+I_{222}}{u_{1}}+\frac{I_{42}-I_{222}}{u_{2}}=I_{4}-I_{22}
$$

At this point we find it expedient to assume a relationship between $u_{1}$ and $u_{2}$, for example $u_{1}=\lambda u_{2}$. For the cube, later on this $\lambda$ shall be taken equal to unity. 
For a specified $\lambda$ we may solve (13) and $u_{1}=\lambda u_{2}$ for $u_{2}$ and $u_{1}$, and then by (10) and (11) determine $a_{1}$ and $a_{2}$. Hence from this point we assume $a_{1}, a_{2}, u_{1}, u_{2}$, as known, and proceed to find $u_{3}, u_{4}, a_{3}, a_{4}$. To this end we rewrite equations (1), (2), (4), and (7) as

$$
\begin{aligned}
8 a_{3}+8 a_{4}=I_{0}-6 a_{1}-12 a_{2} & =p_{0} \\
8 a_{3} u_{3}+8 a_{4} u_{4}=I_{2}-2 a_{1} u_{1}-8 a_{2} u_{2} & =p_{1} \\
8 a_{3} u_{3}{ }^{2}+8 a_{4} u_{4}{ }^{2}=I_{22}-4 a_{2} u_{2}{ }^{2} & =p_{2} \\
8 a_{3} u_{3}{ }^{3}+8 a_{4} u_{4}{ }^{3}=I_{222} & =p_{3} .
\end{aligned}
$$

Now if there is a solution to these equations then there exists a quadratic function $z^{2}+c_{1} z+c_{0}$ such that $u_{3}$ and $u_{4}$ are zeros of the function. Then, however, $c_{1}$ and $c_{0}$ must satisfy

$$
\begin{aligned}
& p_{0} c_{0}+p_{1} c_{1}+p_{2}=0 \\
& p_{1} c_{0}+p_{2} c_{1}+p_{3}=0
\end{aligned}
$$

Assuming such a solution $c_{0}$ and $c_{1}$ we find $u_{3}$ and $u_{4}$

$$
u_{3}=\frac{-c_{1}-\sqrt{c_{1}^{2}-4 c_{0}}}{2}, \quad u_{4}=\frac{-c_{1}+\sqrt{c_{1}^{2}-4 c_{0}}}{2},
$$

and by substitution in $\left(1^{\prime}\right)$ and $\left(2^{\prime}\right)$ we may then find $a_{3}$ and $a_{4}$. It is not simple to prove that (14) is solvable, nor that $u_{3}$ and $u_{4}$ are positive as determined. It is quite conceivable that symmetrical regions exist for which some of these wishes are not fulfilled.

We now let $R$ be the cube of edge 2 , center $(0,0,0)$ and with edges parallel to the axes. Then $I_{0}=8, I_{2}=8 / 3, I_{4}=8 / 5, I_{22}=8 / 9, I_{6}=8 / 7, I_{42}=8 / 15$, $I_{222}=8 / 27$ and we find the following solution if we let $\lambda=1$, i.e., $u_{1}=u_{2}$,

$$
\begin{aligned}
a_{1} & =\frac{1078}{3645} & x_{1} & =\sqrt{\frac{6}{7}} \\
a_{2} & =\frac{343}{3645} & x_{2} & =x_{1}=\sqrt{\frac{6}{7}} \\
u_{3} & =\frac{960-3 \sqrt{28,798}}{2726} & u_{4} & =\frac{960+3 \sqrt{28,798}}{2726} \\
x_{3} & =\sqrt{u_{3}} & a_{3} & =\frac{230-774 u_{3}}{1215\left(u_{4}-u_{3}\right)} \\
x_{4} & =\sqrt{u_{4}} & a_{4} & =\frac{774 u_{4}-230}{1215\left(u_{4}-u_{3}\right)} .
\end{aligned}
$$

The evaluation points all lie in the cube and the weights are positive, both features being desirable for many applications. It is possible to solve the system 
with other specializations. In case one takes $u_{4}=0$ then two 27-point formulas evolve, but some of the points of each lie outside the cube as James ClerkMaxwell found in 1877. The 27-point formulas for the sphere will be given in the sequel. These too, have points outside the sphere.

Let us consider an interpretation of the 34-point formula given here for the cube. Let $f$ be a function and let the formula be applied to approximate the integral over $R$. If one takes any seventh degree polynomial function agreeing with $f$ at the 34 evaluation points, it will have the integral given by the formula no matter how the remaining $120-34=86$ degrees of freedom are used to determine the particular 7 th degree polynomial since there are 120 terms in the general 7 th degree polynomial. These 86 degrees of freedom are available to achieve better error estimates (but not, of course, a smaller error). The formula may be used as a formula for the parallelopipedon by Corollary 1.1.

Error Analysis. In general, error analysis in higher dimensions is much more difficult than for the function of one variable. Nevertheless, it is perhaps of value to summarize briefly one type of error analysis. Let

$$
E(f)=\sum a_{j} f\left(x_{j}\right)-\int_{R} w(x) f(x) d x .
$$

Let $P$ be a class of functions $p$ such that $E(p)=0$. Then $P$ may be considered as a linear space since if $p_{1} \in P$ and $p_{2} \in P$ then $E\left(c_{1} p_{1}+c_{2} p_{2}\right)=0$. Now for any particular $f$ chose $p \in P$ and define $r$ by $f-p=r$. Then

$$
E(f)=E(p+r)=E(r)=\sum a_{j} r\left(x_{j}\right)-\int_{R} w(x) r(x) d x .
$$

This statement holds for all $p \in P$ and every appropriate $f$.

We want to choose $p$ for a given $f$ so that there is a calculable bound to $|E(r)|$ and hence to $|E(f)|$. First choose $p$ so that $p\left(x_{j}\right)=f\left(x_{j}\right)$. Then $r(x)=0$ and $E(r)=-\int_{R} w(x) r(x) d x$ so that $|E(r)| \leq b \int_{R} w(x) d x$ if $|r(x)| \leq b$. The associated approximation problem is to find $p \in P$ so that $p\left(x_{j}\right)=f\left(x_{j}\right)$ and so that $\max |f-p|$ is minimized. If this can be done, we have achieved the best error bound associated with the last inequality. On the other hand, if one takes $p \in P$ such that $\max |f(x)-p(x)| \leq b$ for $x$ ranging over $R$ and any $x$ outside $R$ and if

then

$$
a_{j} \geq 0, \quad \sum a_{j}=\int_{R} w(x) d x
$$

$$
|E(f)| \leq 2 b \int_{R} w(x) d x
$$

In applications the class $P$ does not contain all the functions $p$ such that $E(p)=0$ since these are unknown and essentially unknowable.

Example. Hammer has devised a code for IBM 650 use which has been checked out by Gerald Bartholomew. This code is capable of numerical integration over any symmetrical region in $E_{3}$ using $34,27,14,8$, or 6 points. For a test calculation 
the integral of $1 / \sqrt{x}+1 / \sqrt{y}+1 / \sqrt{z}$, was approximated over the cube with center $(1 / 2,1 / 2,1 / 2)$ and edge 1 . The 34-point formula here described was used and the result was 6.04 compared to the exact value 6 .

Conclusion. We have here summarized a few of the useful theorems for generating numerical integration formulas from given ones, and illustrated one particular approach to obtaining formulas for symmetrical regions. In a sequel we will present all the particular formulas we have calculated for symmetrical regions including some for circle, square, sphere, cube, hypersphere, and hypercube. The method of attack presented for symmetrical regions does not hold much promise of effective extension to arbitrarily high-degree polynomials such as Peirce has carried out for the circular annulus. However, variations and extensions of these methods will be forthcoming.

Preston C. Hammer

A. WAYNE WYMORE

University of Wisconsin

Madison, Wisconsin

This work is supported by a grant of Wisconsin Alumni Research Foundation funds made by the Graduate Research Committee, and by Office of Ordnance Research, U. S. Army contract no. DA-11-022-ORD-2301.

1. J. Clerk-Maxwell, "On approximate multiple integration between limits of summation," Cambridge Phil. Soc., Proc., v. 3, 1877, p. 39-47.

2. G. W. TYLER, "Numerical integration of functions of several variables," Canadian Jn. Math., v. 5, 1953, p. 393-412.

3. P. C. Hammer, O. J. Marlowe \& A. H. Stroud, "Numerical integration over simplexes and cones," $M T A C$, v. 10, 1956, 130-137.

4. P. C. Hammer, \& A. H. Stroud, "Numerical integration over simplexes," $M T A C$, v. 10, 1956, p. $137-139$.

5. W. H. PEIRCE, "Numerical integration over planar regions," Ph.D. Thesis, University of Wisconsin, 1956, available on microfilm.

6. P. Davis \& P. Rabinowitz, "Some Monte Carlo experiments in computing multiple integrals," $M T A C$, v. 10, 1956, p. 1-8.

\section{Solution of Poisson's Equation by Relaxation Method-Normal Gradient Specified on Curved Boundaries}

Introduction. In the application of the relaxation technique to the solution of the Poisson's equation when the normal gradient of the wanted function, $w$, is specified along a curved boundary, difficulty has been experienced in finding a suitable finite difference approximation to the Poisson's equation at nodes that lie adjacent to the curved boundary. It is proposed to present in this article results which will be found adequate for tackling any type of curved boundary in two dimensions.

This problem was treated by $R$. V. Southwell by means of the membrane analogy developed by him [1]. It is, however, preferable to base the approximate representation of differential equations on finite difference theory, which indicates the order of the error involved in the approximation. This has been fully achieved in the case of the Poisson's equation when the boundary condition consists in 